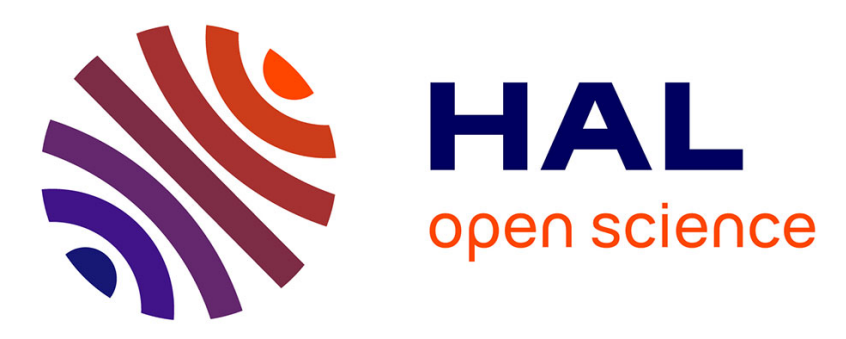

\title{
Electricity Grid Connection of a Tidal Farm: an Active Power Control Framework Constrained to Grid Code Requirements
}

Sofiane Ben Chabane, Mazen Alamir, Mirko Fiacchini, Rachid Riah, Thibaut Kovaltchouk, Seddik Bacha

\section{To cite this version:}

Sofiane Ben Chabane, Mazen Alamir, Mirko Fiacchini, Rachid Riah, Thibaut Kovaltchouk, et al.. Electricity Grid Connection of a Tidal Farm: an Active Power Control Framework Constrained to Grid Code Requirements. IEEE Transactions on Sustainable Energy , 2018, 9 (4), pp.1948-1956. 10.1109/TSTE.2018.2820029 . hal-01753070

\section{HAL Id: hal-01753070 https://hal.univ-grenoble-alpes.fr/hal-01753070}

Submitted on 29 Mar 2018

HAL is a multi-disciplinary open access archive for the deposit and dissemination of scientific research documents, whether they are published or not. The documents may come from teaching and research institutions in France or abroad, or from public or private research centers.
L'archive ouverte pluridisciplinaire HAL, est destinée au dépôt et à la diffusion de documents scientifiques de niveau recherche, publiés ou non, émanant des établissements d'enseignement et de recherche français ou étrangers, des laboratoires publics ou privés. 


\title{
Electricity Grid Connection of a Tidal Farm: an Active Power Control Framework Constrained to Grid Code Requirements
}

\author{
Sofiane Ben Chabane, Mazen Alamir, Mirko Fiacchini, Rachid Riah, \\ Thibaut Kovaltchouk and Seddik Bacha
}

\begin{abstract}
This paper focuses on connecting tidal turbine farms to the French electrical grid. One tidal farm is considered, which is the commercial farm. The connection state is defined by the active power and the voltage at the common coupling point (PCC, also called the delivery point), and is constrained to respect the Grid Code requirements. In order to ensure this connection, this paper develops an active power control framework constrained to the requirements of the Grid Code. This framework is based on the solution of a quadratic programming problem to meet the power demand at the PCC while respecting the requirements of the Grid Code in different plans. As the farm will be installed at the ocean depths, several kilometers of cables will be used to transport the electricity up to the PCC then the power losses are also taken into account in this framework. This framework is applied to a commercial farm with realistic data to show his relevance and effectiveness.
\end{abstract}

Index Terms-Marine energy, Tidal turbine farm, Grid Code, Quadratic programming

\section{INTRODUCTION}

M ARINE energy refers to the energy carried by the ocean waves, tides, salinity and ocean temperature differences. The kinetic energy stored in oceans, that is created by the movement of the water, can be exploited to generate electricity for homes, transports and industries. $70 \%$ of earth surface is covered by oceans, thus the marine energy can be the most important clean energy. This energy can be harvested in many forms, see [1], [2]. In the last years, marine energy technologies and in particular tidal and wave conversion systems have been achieved a considerable progress [3]. European energy system expects that around 2025-2030 the marine energy will play an important role in providing clean energy. This is motivated by the availability of the ocean resources and the technologies that will be matured over time. Tidal turbine is based on nearly the same design and operating principle as the typical wind turbine. Related to the advanced technologies and the current developments on the tidal conversion systems, tidal turbines are considered fully grown to be installed in

S. Ben Chabane, M. Alamir, M. Fiacchini and R. Riah are with Univ. Grenoble Alpes, Gipsa-lab (UMR CNRS 5216), F-38000 Grenoble, France e-mails: benchabanesosso@gmail.com, mazen.alamir@gipsa-lab.grenobleinp.fr, mirko.fiacchini@gipsa-lab.grenoble-inp.fr, rachid.riah88@gmail.com

T. Kovaltchouk and S. Bacha are with Univ. Grenoble Alpes, G2Elab (UMR CNRS 5269), F-38000 Grenoble, France. e-mails: thibaut.kovaltchouk@g2elab.grenoble-inp.fr, seddik.bacha@g2elab.grenobleinp.fr

Manuscript received 05-Sep-2017, Revised and Resubmitted 19-Jan-2018, Accepted for publication 14-Mar-2018 a pre-commercial tidal farms [4], [5], [6]. These tidal farms are considered to be a promising format for industrialization, are similar to offshore wind farms and my benefit from the extensive research and development experience of the wind power industry. A review of the current state of research beyond technology on wave and tidal energies is given in [7].

Tidal turbine farm may have different configurations. Each one gives how the turbines are geographically installed and how the connection between them is established [8]. Transformers are used to step-up voltages in order to enable the connection of the tidal farm to the power grid. Canada and United Kingdom are countries where pre-commercialized prototypes of tidal farm are successfully installed in their maritime areas [9], [10]. Some prototypes are in phases of study in other countries, for examples, Spain [11], Brazil [12], United States [13] and India [14].

In order to preserve power system stability and power quality, Grid Codes for the tidal farm have been revised to integrate requirements from wind turbines farms [15]. In [16], basic understanding and trends for Grid Codes of developed countries in the wind power are presented. The authors analyse the active power control requirements in selected Grid Codes for wind farm. Super Grids projects may include ocean energy and make it a significant source of electricity at the European level. The integration of tidal power into the Grid is still under investigation [17].

Several control strategies for power quality and stability of grid connection of marine energy farm, such as maximum voltage and frequency deviations control, voltage control, active and reactive power control and power factor control are investigated in the literature, see [18] and references therein. For offshore wind farms (being the only marine energy farm already fully operative) the most used schemes of control architecture, in the case of the active power control under Grid Code requirements, are based on the dispatch controller [18], [19]. This controller is used to compute and share the set points (active and reactive power references) to the individual components of the wind power plant. The determination of the individual set points is carried out by using an Optimal Load Flow technique [20].

In this paper, the Grid connection of the tidal turbine farm is examined. The connection state is defined by the active power and the voltage at the common coupling point (PCC, also called the delivery point), and is constrained to respect the Grid Code requirements. In order to ensure this connection an active 
power control framework constrained to the requirements of the Grid Code is developed in this paper. Since the connection of the farm to the Grid requires several kilometers of cables, one novelty here consists in considering the minimisation of the active power losses in the objective of the optimisation problem to be solved. The objective is to distribute the demand on active power at PCC over all the turbines in order to satisfy the active power demand while ensuring the Grid Code requirements. In fact, this is possible by asking each turbine to produce some part from the demand on active power $P^{d}$, which is proportional to its maximum achievable power $\hat{P}_{\ell}^{\max }$. The Grid Code requirements are transformed into constraints, thus the problem consists to optimise a predefined objective function (containing the active power losses) while satisfying a convex set of constraints. Therefore the production of power will be modulated to satisfy the Grid Code requirements and will be in some cases less than the production capacity of the farm.

Preliminary results are obtained by considering the Grid Code requirements in the active/reactive power plan without considering the power losses in the farm [21]. The control is based on the solution of a linear programming problem. The present paper extends the results obtained in [21] by considering, in addition to the Grid Code in the active/reactive power plan, the Grid Code requirements in the voltage/reactive power plan including a primary control of the voltage and the active/reactive power losses of the farm. These power losses are due to cable losses by Joule effect and the presence of transformers in the farm. They have a quadratic form function of the single active/reactive powers of each turbine. In this paper, the solution is given by solving a quadratic programming problem.

This paper is organized as follows. In Section II, the control problem is stated. The feasibility constraints and the Grid Code requirements between the active power and the reactive power are recalled in Section III. Section IV presents the Grid Code requirements in the voltage/reactive power plan, and gives explicitly the details for the requirements on the commercial farm (64 turbines) dictated by the French Distribution Operator System. The expressions of the active and reactive power losses and the constraints formed by all the constraints presented above are given in Section $\mathrm{V}$ and Section VI, respectively. Section VII gives the formulation of the constrained optimization problem and the resulting control framework. The simulations are given in Section VIII for a commercial farm composed by 64 turbines. Finally, concluding results and perspectives are summarized in Section IX.

\section{Problem StATEMEnT}

Figure 1 shows the type of the farm considered in this paper, it has a radial configuration. Assume that this farm contains $n_{h}=64$ turbines. Each 16 turbines represents a hub, then the farm is defined by $n_{h u b}=4$ hubs. The index $\ell \in 1, \cdots, n_{h}$ is used to designate the $\ell^{\text {th }}$ turbine. Before giving the objective of this paper, let us introduce the following previous works:

- The Maximum Power Point Tracking (MPPT) closedloop simulator provided by GENERAL ELECTRIC is used to identify the parameters of the turbine model. This model permits to give the maximum achievable active power as a function of the tidal current for each single turbine. This work is done by following the identification framework given in [22]. The nonlinear relationship to be identified is $\hat{P}_{\ell}^{\max }(t+\tau)=F(\bar{v}(t+\tau))$ where $\tau$ is the sampling period, $\hat{P}_{\ell}^{\max }$ is the prediction of the maximum achievable power and $\bar{v}$ is the vector of past measured wave currents. Note that other identification methods can be used, as least of squares identification [23], [24], maximum of likelihood [25], [26], etc.

- The identification described above requires the availability of the near future wave current over some prediction horizon. This is given by the wave-induced current predictor. This work is given by following the methodology developed in [27].

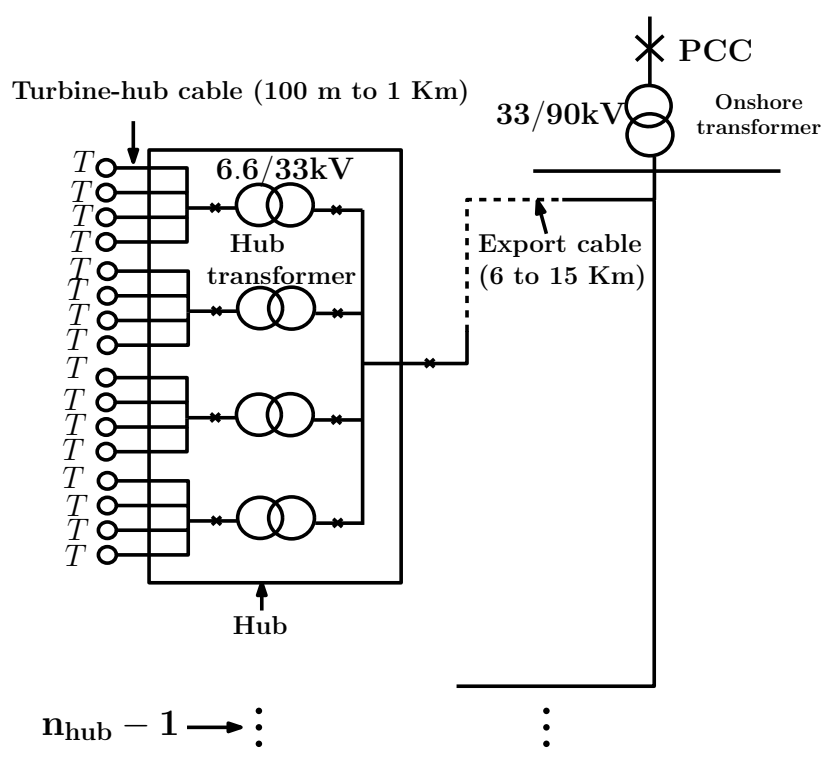

Fig. 1. Farm configuration: radial configuration

Thus in this paper, the predicted value of the maximum achievable active power $\hat{P}_{\ell}^{\max }\left(t+\tau_{c}\right)$ is considered available for a given $\tau_{c}$, where $\tau_{c}$ is the sampling period used in the power prediction model. The maximum achievable active and reactive powers for each turbine is $P_{\ell}^{\max }=1 \mathrm{MW}$ and $Q_{\ell}^{\max }=0.688 \mathrm{MVAr}$, respectively.

The objective of this paper is to guarantee the connection of the tidal farm to an electrical grid. This is done by developing a control architecture of a tidal farm to satisfy the demand in term of active power $P^{d}\left(t+\tau_{c}\right)$ at the PCC, while respecting the Grid Code-related set of constraints in both the plan $(P, Q)$ of active and reactive powers and the plan $(U, Q)$ of the voltage at the PCC and the reactive power. Minimizing the power losses in the farm are also part of the objectives.

In the sequel $P_{\ell}\left(t+\tau_{c}\right)$ and $Q_{\ell}\left(t+\tau_{c}\right)$ will designate the active and reactive powers set-points for the turbine $\ell$ at the future instant $t+\tau_{c}$, such that $P_{\ell}\left(t+\tau_{c}\right) \leq \hat{P}_{\ell}^{\max }\left(t+\tau_{c}\right)$. Therefore, the idea of the control architecture consists to compute the active powers $P_{\ell}$ and the reactive powers $Q_{\ell}$ for all the turbines at each sample time $\tau_{c}$ where the demand 
power $P^{d}\left(t+\tau_{c}\right)$ is assured while respecting the Grid Code constraints in both active/reactive powers $(P, Q)$ plan and voltage/reactive power $(U, Q)$ plan.

\section{ACTIVE/REACTIVE POWER EXPRESSIONS AND CONSTRAINTS}

This section summarizes the constraints on achievable power and grid-like set of constraints in $(P, Q)$ plan (see [21] for details).

The active and reactive powers at the PCC point must satisfy the Grid Code-related constraints. The corresponding constraints can take several forms depending on the country. Fig. 2 illustrates an example of the Grid Code in the $(P, Q)$ plan.

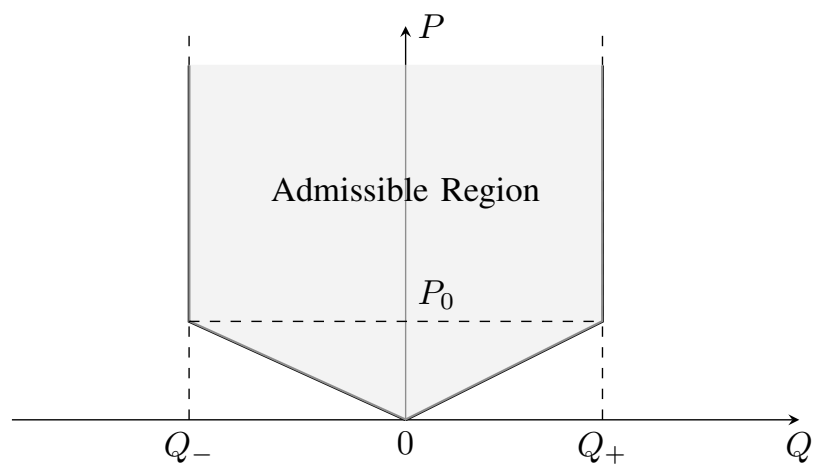

Fig. 2. Example of Grid Code constraints in the $(Q, P)$ plan.

The feasibility constraints are given by

$$
P_{\ell}^{2}+Q_{\ell}^{2} \leq S_{\ell}^{\max 2}
$$

where $S_{\ell}^{\max }$ is the maximum apparent power for the turbine $\ell$, where $\ell=1, \ldots, n_{h}$. These constraints are rewritten in the following condensed form [21]:

$$
\left[A^{(f)}\right] x_{\ell} \leq B_{\ell}^{(f)}
$$

where $A^{(f)}$ and $B^{(f)}$ are given in [21] and $x_{\ell}=\left(P_{\ell}, Q_{\ell}\right)^{\top}$, for $\ell=1, \ldots, n_{h}$.

\section{GRID CODE REQUIREMENTS FOR REACTIVE POWER AND VOLTAGE IN FRANCE}

The voltage is also an essential component for the safety of electrical systems. Maintaining the voltage in predefined ranges is also essential for the proper functioning of receivers and generators of users of the PCC. These receivers or generators can not sustainably accept a voltage significantly deviating from their rated voltage (the tolerance is usually of the order of $\pm 10 \%$ of the rated voltage). Thus, the voltage at the PCC has to be maintained in the so-called normal voltage range. For these reasons, producers of electric power must respect the Grid Code in the $(U, Q)$ plan and regulate the voltage at the PCC.

The producer must satisfy the Grid Code in the $(U, Q)$ plan at the PCC. The constraints are represented by a gray region in Figure 3 (see [28] for more details). The Grid Code can

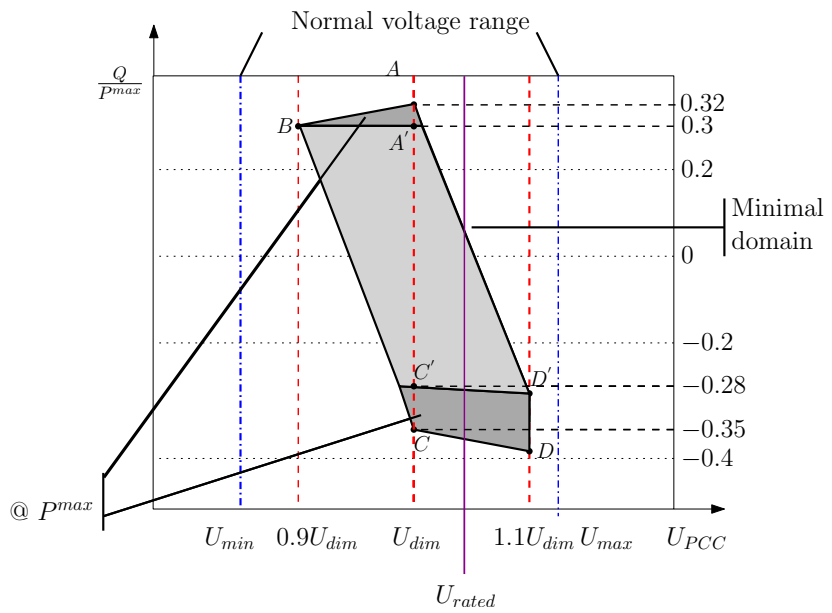

Fig. 3. $[U, Q]$ requirements for generators with two domain: $P=P^{\max }$ and $P<P^{\max }$

depend on the rated voltage value at the PCC or the capacity value of the plant. In addition to the requirements in the $(U, Q)$ plan, the producers of electrical power must contribute to the primary control of the voltage. The producer that contributes to the voltage primary control must have the capacity of providing and absorbing the reactive power required. In the case of the commercial farm, the voltage at the PCC has to be regulated to a set-point value $U_{\text {cons }}$ varying linearly as a function of the reactive power $Q$ with an adjustable slope $\lambda$, and defined by

$$
U_{P C C}+\lambda Q=U_{\text {cons }},
$$

$U_{\text {cons }}$ and $\lambda$ are given by the french transmission system operator RTE (Réseau de Transport d'Electricité). $\lambda=\frac{\lambda^{\prime} U_{\text {rated }}}{Q^{\text {max }}}$, where $0.02 \leq \lambda^{\prime} \leq 0.07$ and $Q^{\max }$ is the maximum reactive power attainable by the farm, i.e. $Q^{\max }=0.688 \times n_{h}$ MVAr. $U_{P C C}$ is replaced by its value given in Appendix A (equation (30)). In this appendix, $U_{P C C}$ is function of $P, Q, U_{r}$ (the grid voltage), $\Psi$ (the angle of the grid impedance) and $S_{k}$ (the apparent power of the grid). Recall that $U_{r}$ is known and viewed as an exogenous input (Appendix A).

\section{A. Constraints in the $(U, Q)$ plan}

There are two types of constraints: equality constraints from the primary voltage control and inequality constraints from the Grid Code in the $(U, Q)$ plan.

Equality constraints: From the equation (3) and taking into account the value of $U_{P C C}$ in (30), we have:

$$
U_{\text {cons }}=U_{r}+\frac{P \cdot \cos (\Psi)+Q \cdot \sin (\Psi)}{S_{k}} U_{r}+\lambda Q .
$$

Inequality constraints: The requirements for generators in the $(U, Q)$ plan depends on the value of the active power $P$, see Figure 3. The couple $\left(U_{P C C}, \frac{Q}{P^{\max }}\right)$ has to be in the light gray set when $P<P^{\max }$ and in both light and dark gray sets when $P=P^{\max }$.

Remark 1: The control equation of the voltage (4) can be violated when the Grid Code requirements in Figure 3 
are saturated. For this reason, this equality is relaxed by introducing two scalar variables $\alpha_{1}$ and $\alpha_{2}$ :

$$
\begin{aligned}
U_{\text {cons }}= & U_{r}+\frac{P \cdot \cos (\Psi)+Q \cdot \sin (\Psi)}{S_{k}} U_{r}+\lambda Q+ \\
& \alpha_{1} \lambda Q^{\text {max }}-\alpha_{2} \lambda Q^{\text {max }},
\end{aligned}
$$

where $\alpha_{1} \geq 0$ and $\alpha_{2} \geq 0$ have to be penalized in the objective function that will be given in the sequel.

\section{ACTIVE AND REACTIVE POWER LOSSES}

For an electric part of the farm, the reactive power injected at one end will be the reactive power at the other end plus the reactive power produced or absorbed by the electric part. In this paper, the reactive power loss is the loss in the amplitude of the instantaneous reactive power. Thus, the farm presents power losses in active and reactive powers. These losses are caused by different parts of the farm: Turbine-hub cables, export cables, turbines transformers, hub transformers and Onshore transformer.

The active power consumed by a cable $\mathcal{C}$ is given by

$$
P_{\mathcal{C}}=r L \frac{S^{2}}{U^{2}}
$$

where $r$ is the linear resistance of the cable, $L$ is its length, $S$ the apparent power $\left(S^{2}=P^{2}+Q^{2}\right)$ and $U$ the voltage in this cable.

The reactive power produced or absorbed by a cable $\mathcal{C}$ is given by

$$
Q_{\mathcal{C}}=-c \omega L U^{2}+l \omega L \frac{S^{2}}{U^{2}}
$$

where $c$ is the linear capacitance, $l$ the linear inductance and $\omega$ is the the pulsation of the voltage.

The active power consumed by a transformer is given by

$$
P^{\text {trans }}=\frac{1}{R_{F}} U_{1}^{2}+r_{c} \frac{S^{2}}{U_{2}^{2}},
$$

where $R_{F}$ are iron losses resistance, $r_{c}$ are copper losses resistance, $U_{1}$ and $U_{2}$ are the primary and secondary voltage of the transformer.

The reactive power produced or absorbed by a transformer is given by

$$
Q^{\text {trans }}=\frac{1}{L_{M} \omega} U_{1}^{2}+l_{f} \omega \frac{S^{2}}{U_{2}^{2}} .
$$

In order to compensate a lack of reactive power, a FACTS (Flexible Alternating Current Transmission System) will be potentially used at the connection point (PCC). This FACTS is a pure reactive power producer. It produces $Q_{F A C T S} \mathrm{VAr}$.

The vector of decision variables $X$ is given by

$$
X=\left(\begin{array}{llllllll}
x_{1}^{\top} & \ldots & x_{n_{h}}^{\top} & \gamma^{d} & \alpha_{1} & \alpha_{2} & Q_{F A C T S} & \gamma
\end{array}\right)^{\top},
$$

where $X \in \mathbb{R}^{2 n_{h}+5}, x_{\ell}=\left(P_{\ell}, Q_{\ell}\right)^{\top}$, for $\ell=1, \ldots, n_{h}$ with $\gamma \geq 0$ and $\gamma^{d} \geq 0$ defined in Section VII.

Given the radial configuration of the farm in Figure 1 and using Boucherot's theorem [29], the total active and reactive power losses consumed, produced or absorbed, respectively, by the farm are a quadratic function of $P_{\ell}$ and $Q_{\ell}$, for $\ell=1, \ldots, n_{h}$ and are denoted $P_{\text {loss }}(X)$ and $Q_{\text {loss }}(X)$, respectively. Note that the maximum of these powers $P_{\text {loss }}^{\max }$ and $Q_{\text {loss }}^{\max }$ can be easily computed.

\section{REWRITING OF ALL CONSTRAINTS}

Taking into account the power losses, all the constraints in both $(P, Q)$ plan and $(U, Q)$ plan have to be reconsidered by using

$$
\begin{aligned}
P & =\sum_{\ell=1}^{n_{h}} P_{\ell}-P_{\text {loss }}, \\
Q & =\sum_{\ell=1}^{n_{h}} Q_{\ell}+Q_{F A C T S}-Q_{\text {loss }} .
\end{aligned}
$$

Note that the active and reactive power losses, $P_{\text {loss }}$ and $Q_{\text {loss }}$, will be estimated on-line. The details of this estimation are given in the next section.

Thus, the Grid Code in the $(P, Q)$ plan, represented in Figure 2, can be written in the following form:

$$
A_{G C-P Q}^{i n e q} X \leq B_{G C-P Q}^{\text {ineq }}\left(P_{\text {loss }}, Q_{\text {loss }}\right)
$$

Replacing $U_{P C C}$ by its value which is given by (30), the requirements for the Grid Code in the $(U, Q)$ plan, represented in Figure 3 and the control equation of the voltage given by (5), are equivalent to:

$$
A_{G C-U Q}^{i n e q} X \leq B_{G C-U Q}^{i n e q}\left(P_{l o s s}, Q_{l o s s}\right) .
$$

$$
A_{G C-U Q}^{e q} X=B_{G C-U Q}^{e q}\left(P_{l o s s}, Q_{l o s s}\right) .
$$

Remark 2: $A_{G C-P Q}^{\text {ineq }}, B_{G C-P Q}^{\text {ineq }}, A_{G C-U Q}^{e q}, B_{G C-U Q}^{e q}$, $A_{G C-U Q}^{i n e q}$ and $B_{G C-U Q}^{i n e q}$ are easy to obtain but not explicitly given here, in order to simplify the reading of this paper.

In the next section, the formulation of the constrained optimization problem will be presented.

\section{DESIGN OF THE CONTROL FARM}

In this section, all the constraints on the active and reactive powers $\left\{x_{\ell}\right\}_{\ell=1}^{n_{h}}$, the reactive power $Q_{F A C T S}$ and the relaxation parameters $\alpha_{1}$ and $\alpha_{2}$ (introduced for the voltage primary control in Remark 1) will be gathered. The active power $P_{\ell}$ to be produced by each turbine $\ell$ is uniformly distributed with respect to the predicted maximum active power $\hat{P}_{\ell}^{\max }$ through the parameter $\gamma^{d}$ (equation (16)) which becomes a decision variable. A parameter $\gamma$ is added to the power demand equation (19). This parameter permits to ensure the feasibility of the optimization problem for each profile of the power demand $P^{d}$. The objective function to be minimized is defined in equation (23). So, the problem to solve is a quadratic programming problem that will be defined below. 


\section{A. Active power for each turbine}

Each turbine $\ell$ produces an active power $P_{\ell}$ :

$$
P_{\ell}=\left(1-\gamma^{d}\right) \hat{P}_{\ell}^{\max },
$$

where $\gamma^{d}$ determines the ratio between $P_{\ell}$ and $\hat{P}_{\ell}^{\max }$.

Each turbine in the farm may be off-line for different reasons, for example maintenance or accident. Thus in this work the distribution vector $\bar{\pi}$ is introduced. It is defined as follows:

$$
\bar{\pi}:=\left(\begin{array}{c}
\pi_{1} \\
\vdots \\
\pi_{n_{h}}
\end{array}\right) \in[0,1]^{n_{h}} .
$$

The parameters $\pi_{\ell}$ may take value in $[0,1] . \pi_{\ell}=0$ means that some turbines are declared to be off-line. Generally, it can be used to permit the non uniform distribution of the load associated to each turbine. This can be interpreted as the manner to consider the current state of the health of the individual turbines. By considering these parameters the equality constraint (16) becomes

$$
P_{\ell}=\left(1-\gamma^{d}\right)\left[\pi_{\ell} \hat{P}_{\ell}^{\max }\right],
$$

with $\gamma^{d} \in[0,1]$.

\section{B. Desired power}

In this paper the control objective of the active power control framework is to well satisfy the power demand $P^{d}$ at the PCC. This can be formulated by

$$
P=(1-\gamma) P^{d},
$$

where $\gamma \geq 0$ has to be heavily penalized. This parameter is a relaxation and can be different from 0 when the power demand $P^{d}\left(t+\tau_{c}\right)$ is greater than the maximum achievable power $\hat{P}^{\max }\left(t+\tau_{c}\right)$. Thus, equation (19) is equivalent to

$$
\sum_{\ell=1}^{n_{h}} P_{\ell}=(1-\gamma) P^{d}+\hat{P}_{l o s s} .
$$

\section{Tidal farm optimization problem}

The global optimization problem is formulated using the vector of decision variables $X$ which is given in (10). By using the prediction of the maximum achievable power of each turbine, $\hat{P}_{\ell}^{\max }$, the inequality constraints for the commercial farm, gathering (2), (13), (14) while considering the fact that $P_{\ell} \leq 1 \mathrm{MW}$ and $Q_{\ell} \leq 0.688 \mathrm{MVA}$, can be put in the following form:

$$
A_{\text {ineq }} X \leq B_{\text {ineq }}\left(P_{\text {loss }}, Q_{\text {loss }}\right) .
$$

In the same way, the equality constraints (15), (18) and (20) can also be rewritten in this linear form on $X$ :

$$
A_{e q} X=B_{e q}\left(P_{l o s s}, Q_{l o s s}\right) \text {. }
$$

Finally, the objective function to be minimized is:

$$
C(X)=c_{1} \frac{P_{\text {loss }}(X)}{P_{\text {loss }}^{\text {max }}}+c_{2} \gamma+\gamma^{d}+c_{3}\left(\alpha_{1}+\alpha_{2}\right),
$$

where $P_{\text {loss }}(X)$ is quadratic in $X$ (see Section V) and $c_{2} \gamma+$ $\gamma^{d}+c_{3}\left(\alpha_{1}+\alpha_{2}\right)$ is linear in $X$.

By manipulating the objective function, the optimization problem can be rewritten in the following quadratic programming form:

$$
\begin{array}{ll}
\min _{X} & {\left[\frac{1}{2} X^{\top} H X+f^{T} X\right]} \\
\text { s.t. } & \\
& A_{\text {ineq }} X \leq B_{\text {ineq }}\left(P_{\text {loss }}, Q_{\text {loss }}\right), \\
& A_{\text {eq }} X=B_{\text {eq }}\left(P_{\text {loss }}, Q_{\text {loss }}\right) .
\end{array}
$$

The previous works, reminded in Section II, have as results the near future wave current over some prediction horizon, $\bar{v}\left(t+\tau_{c}\right)$, and the prediction of the maximum achievable power of each turbine, $\hat{P}^{\max }\left(t+\tau_{c}\right)$. Therefore, the optimization problem given in this paper has to be solved for all the future instants of the form $t+i \tau_{c}$. Thus the active and reactive powers set-points for the turbines will be computed at all these future instants.

The optimisation problem (24) is hard to solve since the constraints depend on the active and reactive power losses $P_{l o s s}$ and $Q_{\text {loss }}$ where these power losses depend on the active and reactive powers, $P_{\ell}$ and $Q_{\ell}, \quad \ell=1, \ldots, n_{h}$, see Section $\mathrm{V}$. The $P_{\ell}$ and $Q_{\ell}, \quad \ell=1, \ldots, n_{h}$, are part of the decision variable $X$ of the optimisation problem (24). The power losses $P_{\text {loss }}$ and $Q_{\text {loss }}$ are quadratic in $X$. In order to keep the problem simple, the estimation of $P_{\text {loss }}$ and $Q_{\text {loss }}$ is proposed. The practical solution is done by formulating the problem as a fixed-point problem of an implicit function which results from the solution of a mathematical programming problem (24). It is summarized in the following algorithm:

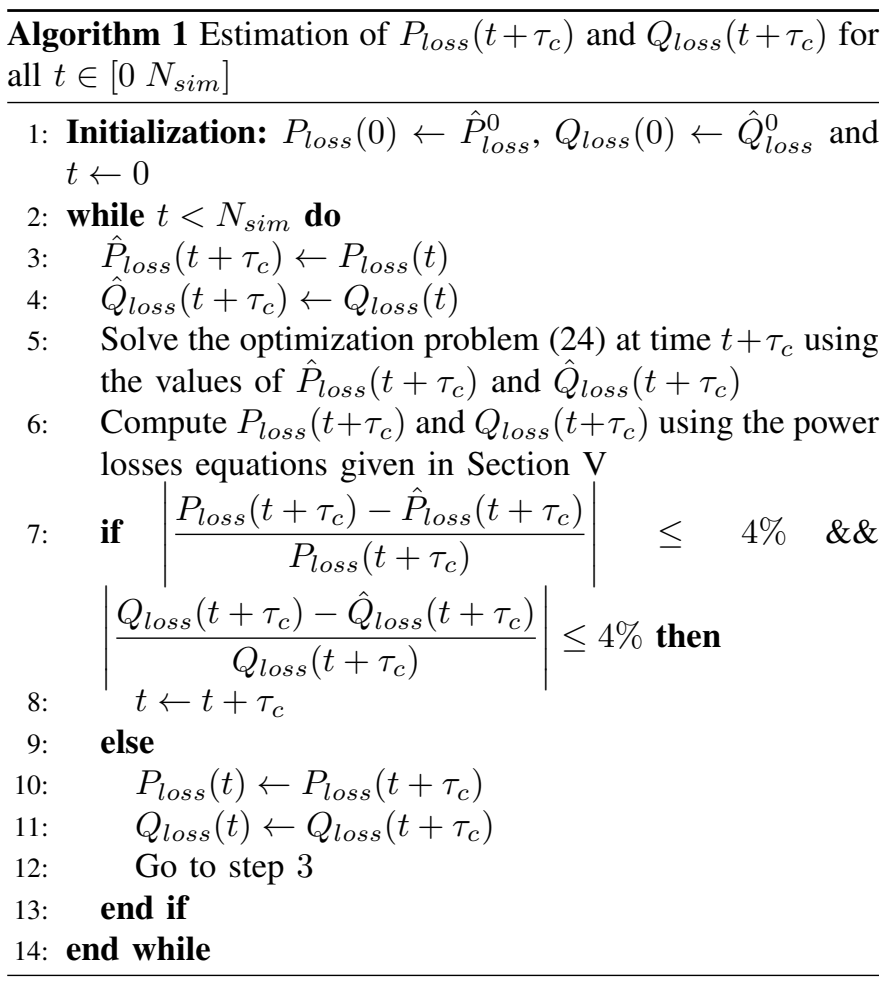

This means that, at each sample time $\tau_{c}$, the active and reactive power losses are readjusted until a good accuracy 
(4\%) is reached. Note that $N_{\text {sim }}$ represents the simulation time and $\hat{P}_{\text {loss }}^{0}$ and $\hat{Q}_{\text {loss }}^{0}$ have to be predefined for the initialization of the algorithm.

\section{Simulation RESUltS}

The active power control framework developed in this paper will be tested in the case of the commercial farm. This farm is defined by 64 turbines which are divided into 4 hubs. The commercial farm has a radial configuration. In this section two examples will be shown for the commercial farm. Here the commercial farm with FACTS is considered. The parameters of the objective function (23) are $c_{1}=1, c_{2}=10^{2}$ and $c_{3}=$ $10^{2}$.

In order to generate the time series of individual achievable powers $\hat{P}_{\ell}^{\max }$, for $\ell=1, \ldots, n_{h}$, an integer $N_{\text {saut }}=10$ is used as well as the translation of one time series defined in [21]. This is done as follow:

$$
\hat{P}_{\ell}^{\max }\left(t+i \tau_{c}\right):=\hat{P}_{0}^{\max }\left(t+\left(i+(\ell-1) N_{\text {saut }}\right) \tau_{c}\right) .
$$

In fact the spatial dispersion of the tidal current is emulated with the previous consideration. The profile of $\hat{P}_{0}^{\max }$ chosen in this simulation is generated randomly according to the realistic data of the turbine.

In the real context, the local tidal current will be predicted locally for each turbine. Therefore, once the maximum achievable power $\hat{P}_{\ell}^{\max }$ is calculated for each turbine, it is to be sent to the central computation unit of the tidal farm.

The values of the apparent powers, $S_{\ell}^{\max }, \ell \in 1, \cdots, n_{h}$, in equation (1), have been taken equal to $\gamma_{S}:=1.05$ times the maximum achieved active power. They are given as follow:

$$
S_{\ell}^{\max }:=\gamma_{S} \pi_{\ell} \hat{P}_{\ell}^{\max }, \quad \ell \in 1, \cdots, n_{h} .
$$

In the French Grid Code $(P, Q)$, the value $P_{0}$ used to define the admissible region of the couple of active and reactive powers, $(P, Q)$, is computed by the following equation:

$$
P_{0}=\gamma_{P_{0}} \times n_{h} \times \max _{\ell=1, \cdots, n_{h}}\left[S_{\ell}^{\max }\right], \gamma_{P_{0}}=0.4 .
$$

The limits of the reactive power, $Q^{+}$and $Q^{-}$, implied in the definition of this Grid Code are given by:

$$
\begin{aligned}
& Q^{+}=\gamma_{Q^{+}} \times P_{0}, \quad \gamma_{Q^{+}}=0.3, \\
& Q^{-}=-\gamma_{Q^{-}} \times P_{0}, \quad \gamma_{Q^{-}}=0.3 .
\end{aligned}
$$

The French Grid Code parameters in the $(U, Q)$ plan are as follows. The sizing voltage $U_{\text {dim }}=89 \mathrm{kV}$. The commercial farm $(64 \cdot 1=64 \mathrm{MW})$ is connected to the transmission system HTB1 voltage. Then the normal voltage range is defined by $U_{\text {max }}=100 \mathrm{kV}, U_{\text {min }}=78 \mathrm{kV}$ and $U_{\text {rated }}=90 \mathrm{kV} . U_{r}=$ $U_{\text {cons }}, \tan (\Psi)=X / R=10$ and $S_{k}=1200$ MVA. Note that the grid voltage $U_{r}$ here is chosen for the simulation. In the real case, $U_{r}$ is given by RTE at each sample time. $\lambda^{\prime}=4 \%$ and $Q^{\max }=30 M V A r$.

The cable length between the PCC and the hub is considered as equal to $12 \mathrm{Km}$. Random draw has been used to choose the distance between each turbine and the hub in the interval $[100 m, 1000 m]$.
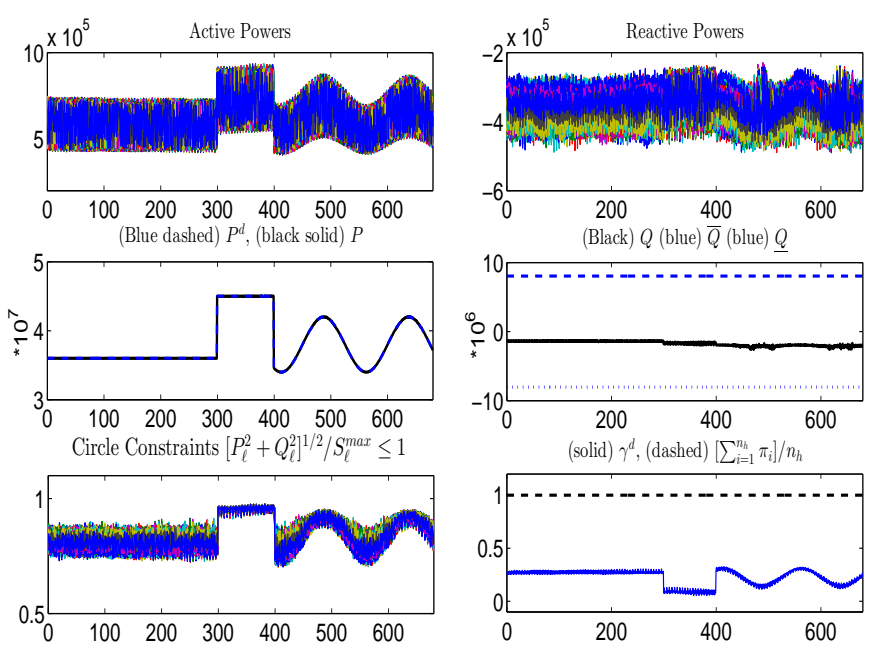

Fig. 4. Saturation of the Grid Code $(U, Q)$ in the increasing sense of the axis $U$ (Example 1). The axis labels of the sub-figures are time in second.

\section{A. Example 1}

The voltage $U_{\text {cons }}$ is taken equal to $90 \mathrm{kV}$, $\left(U_{\text {cons }}=90 \mathrm{kV}\right)$, during the interval time $[0 s, 400 s]$ which is the nominal value, while this value is set to $94 \mathrm{kV}$ for the interval time $[400 s, 680 s]$. The value of $94 \mathrm{kV}$ will show the saturation behaviour of the farm Grid Code requirements in the $(U, Q)$ plan. Figure 4 represents the active and reactive powers, $\alpha_{1}$
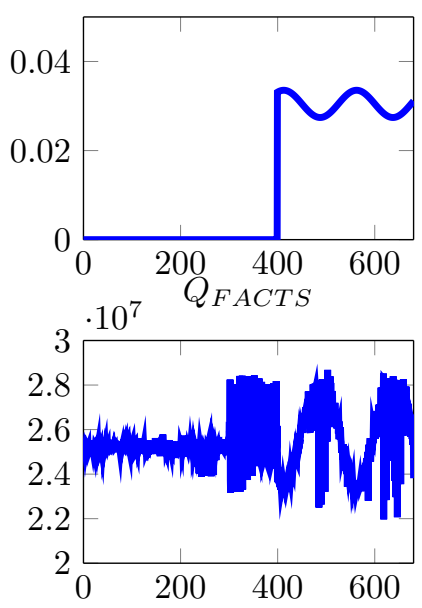

$\alpha_{2}$
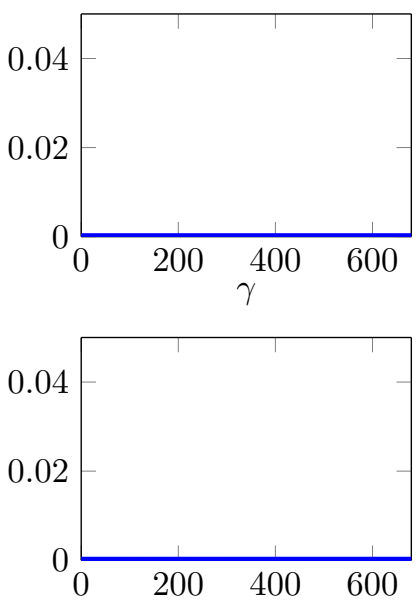

Fig. 5. $\alpha_{1}, \alpha_{2}, \gamma$, and $Q_{F A C T S}$ values (Example 1). The axis labels of the sub-figures are time in second.

the produced active power $P$ and the desired power $P^{d}$, the Grid Code constraints in the $(P, Q)$ plan (where, $\bar{Q}=$ $\min \left\{1, \frac{P}{P_{0}}\right\} \times Q^{+}$is the upper bound of the reactive power $Q$ and $\underline{Q}=\min \left\{1, \frac{P}{P_{0}}\right\} \times Q^{-}$is the lower bound of the reactive power $Q$ ), the feasibility constraints (1) and the value of $\gamma^{d}$. All the constraints are satisfied.

Figure 5 represents the values of the relaxation parameters $\alpha_{1}$ and $\alpha_{2}$ introduced in (5) for the voltage control equation, the variation of the reactive power $Q_{F A C T S}$ produced by the FACTS and the parameter $\gamma$. Notice that the parameters $\alpha_{1}$ 


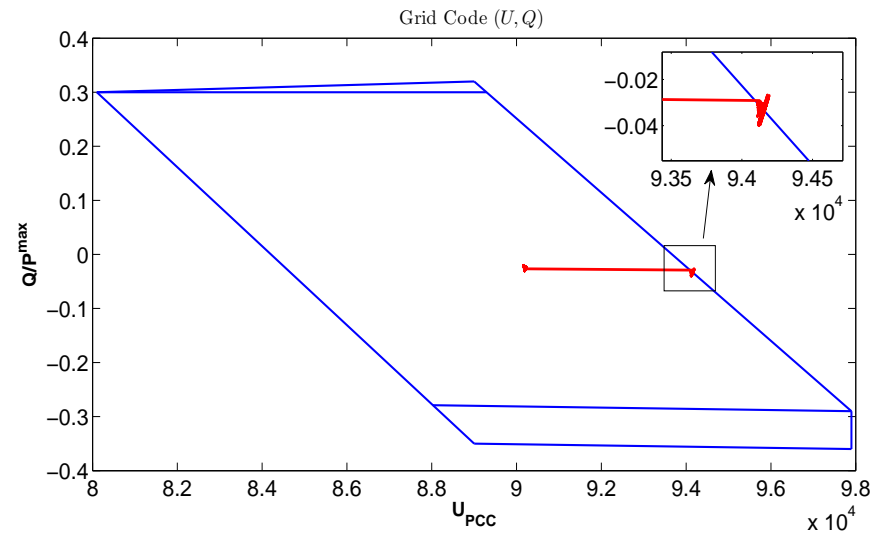

Fig. 6. $[U, Q]$ diagram (Example 1).

and $\alpha_{2}$ are equal to 0 for the nominal conditions (interval time $[0 s, 400 s]$ ) while the parameter $\alpha_{1}$ is positive when the Grid Code in the $(U, Q)$ plan is saturated in the growing sense of the axis $U$ (interval time $[400 s, 680 s]$ ), see Remark 1 .

The admissible domain of the voltage and the reactive power $(U, Q)$ at the PCC, defined by the Grid Code requirements, is represented in Figure 6. We see that the variation of $U_{P C C}$ function of $\frac{Q}{P^{\max }}$, represented in red, is in the limits of the domain, which prove that the Grid Code requirement is satisfied. This is due to the value of $U_{\text {cons }}=94 \mathrm{kV}$. The small violations are due to the estimation errors of the active and reactive power losses.

\section{B. Example 2}

In this example, the voltage $U_{\text {cons }}$ is taken equal to $90 \mathrm{kV}$, $\left(U_{\text {cons }}=90 \mathrm{kV}\right)$, during the interval time $[0 s, 400 s]$, while this value is set to $84 \mathrm{kV}$ for the interval time [ $400 s, 680 s]$. Low values of $U_{\text {cons }}$ comparing to Example 1 in the interval time $[400 s, 680 s]$ are taken and in some intervals time the power demand $P^{d}$ exceeds the maximum achievable power $\hat{P}^{\max }$. The parameter $\alpha_{2}$ given in Remark 1 will be activated due to the low value of $U_{\text {cons }}=84 \mathrm{kV}$ in interval time $[400 \mathrm{~s}, 680 \mathrm{~s}]$. The parameter $\gamma$ introduced in (19) will also be activated because $P^{d}$ exceeds $\hat{P}^{\max }$.

In Figure 7, we remark that the power demand $P^{d}$ is greater that the maximum achievable power during interval time $[450 s, 520 s]$ and interval time $[605 s, 670 s]$. During this period, the turbines produce the maximum of power which is confirmed by the value of $\gamma^{d}=0$ in interval time $[450 s, 520 s]$ and interval time $[605 s, 670 s]$. This saturates the feasibility constraints (1) (circle constraints) due the important values of $P_{\ell}$. This is also confirmed by the value of $\gamma$, which is different from 0 during this period, as shown in Figure 8. In this figure, the parameter $\alpha_{2}$, introduced in (5), is positive when the Grid Code $(U, Q)$ is saturated in the decreasing sense of the axis $U$ as shown in Figure $9\left(U_{\text {cons }}=84 \mathrm{kV}\right.$ in the interval time $[400 s, 680 s])$. This finding is stated in Remark 1. The small violations in the $(U, Q)$ plan are due to the estimation errors of the active and reactive power losses.

Notice that particulate cases, where some turbines are off-line during some interval times, are considered in this
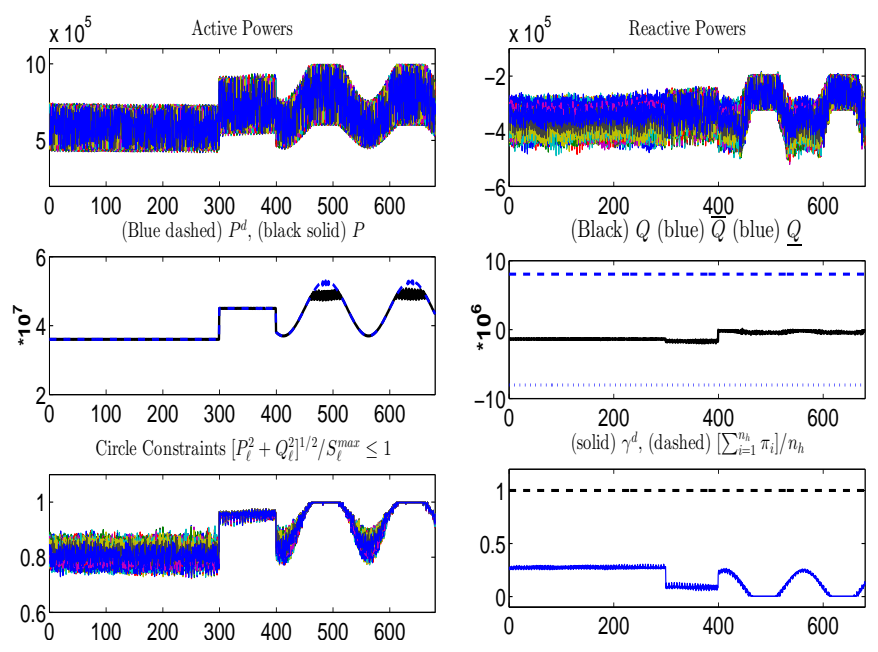

Fig. 7. Saturation of the Grid Code $(U, Q)$ in the decreasing sense of the axis $U, P^{d}$ exceeds $\hat{P}^{\max }$ in some intervals time (Example 2 ). The axis labels of the sub-figures are time in second.
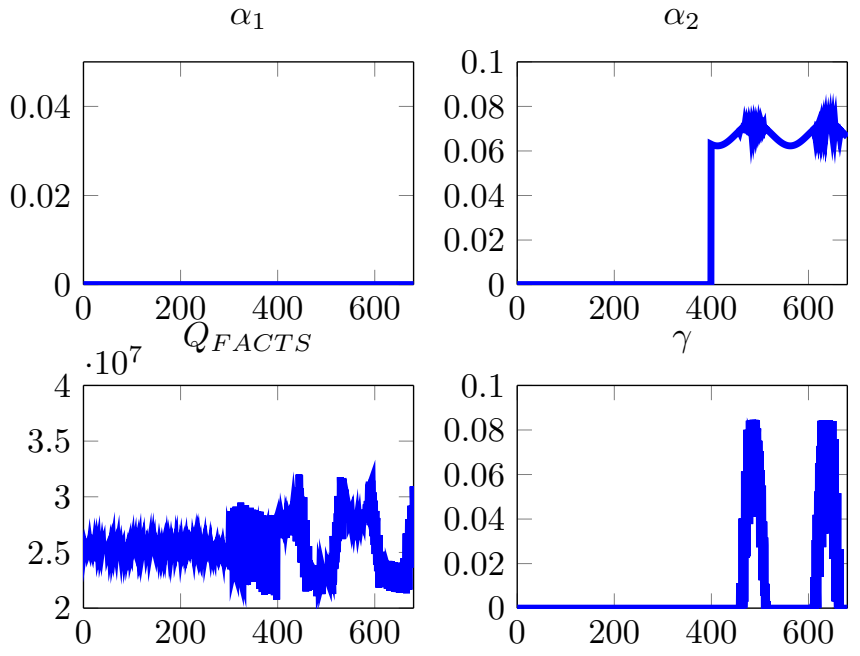

Fig. 8. $\alpha_{1}, \alpha_{2}, \gamma$, and $Q_{F A C T S}$ values (Example 2). The axis labels of the sub-figures are time in second.

framework. In fact, the controller is designed in order to adapt itself when some turbines are off-line. The active power control framework developed in this paper is also applied for the commercial farm without FACTS and the results are quite similar to the results shown above for the commercial farm with FACTS. The difference is in the values of the reactive powers $Q_{\ell}$ which are greater than the values in the case with FACTS since the turbines instead of the FACTS have to generate the appropriate reactive power. The use of FACTS located a short distance away from the PCC permits to the farm to maximize its active power production. In the optimization methods, this enables more flexibility in order to respect all the farm constraints.

\section{CONCLUSION}

In this paper, an active power control framework for a tidal turbine farm has been developed. This framework guarantees the connection of the farm to the French electrical grid. 


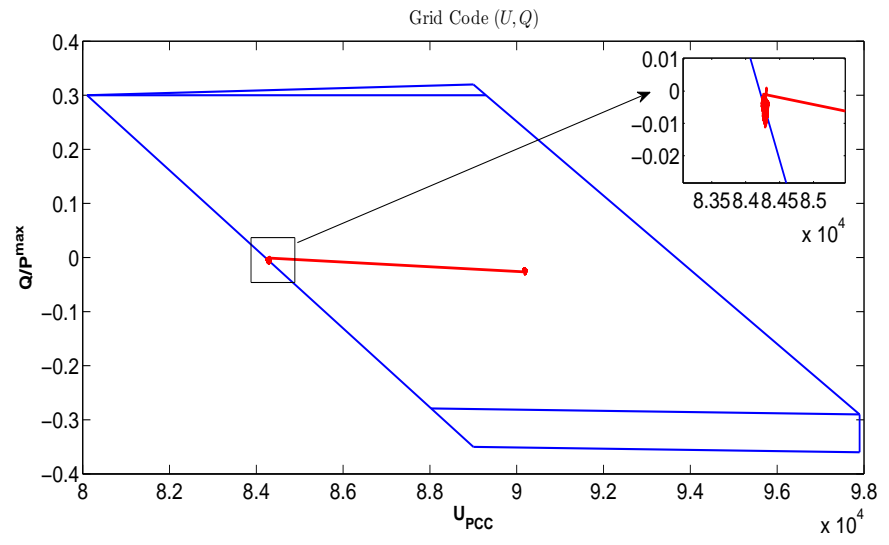

Fig. 9. $[U, Q]$ diagram (Example 2).

The feasibility constraints for each turbine, Grid Code in the $(P, Q)$ plan and $(U, Q)$ plan are respected considering the power losses in the farm. The proposed controller permits to distribute the active power demand at the PCC over all the turbines by asking each turbine to produce an active power which is proportional to its maximum achievable active power. Two examples are provided to show the effectiveness of this controller on the commercial farm (64 turbines). This controller can be applied for other farms, as a pilot farm (4 turbines) by introducing small modifications on the Grid Code in the $(U, Q)$ plan. This is due to the connection of the pilot farm to the distribution system operator contrary to the commercial farm that is connected to the transport system operator. These constraints will have also the same form as in the commercial farm. Three important perspectives can be released. The first one is to take into account the variation of the different voltages in the farm. This point was undertaken and gave good results and is still in progress. The second perspective is to consider a control of the frequency $f$, by considering a variable $f$. The third is to evaluate and discuss the communication structure between the central computational unit and the individual turbines.

\section{APPENDIX A}

The grid at the PCC can be represented with an equivalent grid impedance $Z=|Z| e^{j \Psi}=R+j X$, with $\Psi$ the angle of the grid impedance (see Figure 10). If we note $I$ the current from the farm and $\phi$ the angle between the current and the grid voltage $U_{r}$, we write that:

$$
P=\sqrt{3} \cdot U_{r} \cdot I \cdot \cos (\phi), \quad Q=\sqrt{3} \cdot U_{r} \cdot I \cdot \sin (\phi) .
$$

Note that $U_{r}$ is measured by RTE, with a good precision, at each sample time. So, $U_{r}$ is known and is viewed as an exogenous input. The voltage variation at the PCC due to the active and reactive powers of the farm is given by:

$$
\Delta U_{r}=R \cdot I \cdot \cos (\phi)+X \cdot I \cdot \sin (\phi) .
$$

This can be rewritten as a relative voltage variation

$$
\frac{\Delta U_{r}}{U_{r}}=\frac{R \cdot \sqrt{3} \cdot U_{r} \cdot I \cdot \cos (\phi)+X \cdot \sqrt{3} \cdot U_{r} \cdot I \cdot \sin (\phi)}{\sqrt{3} \cdot U_{r}^{2}} .
$$

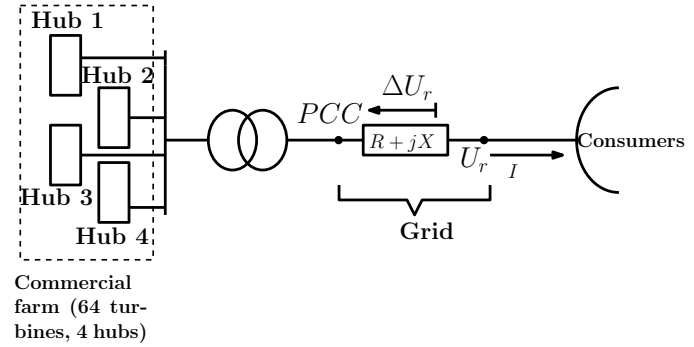

Fig. 10. Voltage variation at the PCC

If we note the apparent power by $S_{k}=\sqrt{3} U_{r}^{2} /|Z|$, then

$$
\frac{\Delta U_{r}}{U_{r}}=\frac{P \cdot \cos (\Psi)+Q \cdot \sin (\Psi)}{S_{k}} .
$$

Thus, the voltage at the connection point is given by

$$
U_{P C C}=U_{r}+\frac{P \cdot \cos (\Psi)+Q \cdot \sin (\Psi)}{S_{k}} U_{r} .
$$

\section{ACKNOWLEDGEMENT}

This work has been supported by the PRISMER project, a project which received the support of the French National Environment and Energy Management Agency (ADEME) in the frame of the Investments for the Future Programme (Programme d'Investissements d'Avenir, PIA). The coordinator of this project is GE, and its purpose is the tidal turbines subsea electrical interconnection and power transmission from a farm. The investment in this project is $21 \mathrm{M} €$ for a duration of 4 years. Aside from GE, the other members of this project are: Engie, Silec Cable, Jifmar Offshore Services, Sector group, G2Elab and Gipsa-lab.

\section{REFERENCES}

[1] J. Huckerby, H. Jeffrey, and B. Jay, "An international vision for ocean energy," in Ocean energy systems implementing agreement, 2011.

[2] E. Brito, A. Melo, and J. Vilatte, "Annual report 2014. implementing agreement on ocean energy systems. IEA-OES," in http://dx.doi.org/10.1017/S000197200000176, 2015.

[3] R. Charlier, "Ocean energies: environmental, economic, and technological aspects of alternative power sources," Elsevier publication, 1993, 1993.

[4] EDF, "Tidal farm of Paimpol-Bréhat," 2004, france.

[5] R. project, "Verdant power," in http://www.verdantpower.com, 2002, new York.

[6] D. Magagna, A. MacGillivray, H. Jeffrey, C. Hanmer, and A. BadcockBroe, "Wave and tidal energy strategic technology agenda," in Strategic initiative for ocean energy (SI ocean), 2014.

[7] A. Uihlen and D. Magagna, "Wave and tidal current energy - a review of the current state of research beyond technology," Renewable and Sustainable Energy Reviews, vol. 58, pp. 1070-1081, 2016.

[8] Y. Li, B. J. Lence, and S. M. Calisal, "Modeling a tidal turbine farm system with vertical axis tidal turbine," in IEEE International Conference on Systems, Man, and Cybernetics (SMC 2007), 2007, montral, QC, Canada.

[9] P. Fraenkel, "Power from marine currents," Proceedings of Istitution of Mechanical Engineers, vol. 216, pp. 1-14, 2002.

[10] A. Cornett, "Inventory of canada's marine renewable energy ressources," NRC-CHC Technical Report-041, 2006,, 2006, ottawa, Canada.

[11] L. T. et al., "Tides and tidal energetics of the Strait of Gibraltar (Spain) a modelling approach," Tectonophysics 294, 1998, 1998.

[12] S. Anderson, "Tide-generated energy at the amazon estuary:the use of traditional technology to support modern development," Renewable Energy, vol. 3(2), pp. 271-278, 1998. 
[13] R. Bedard, "North America Tidal In Stream Energy Conversion Technology Feasibility Study," ERPI Report TP008, 2006, 2006.

[14] D. Deokar, "Modeling tidal power plant at sephale," IEEE Region 10 Annual International Conference, Proceedings/TENCON, vol. 2, pp. 2278-2283, 1999.

[15] A. Blavette, A. W. Lewis, D. L. O'Sullivan, and M. G. Egan, "Grid integration of wave and tidal energy," Proccedings of the $30^{\text {th }}$ International Conference on Ocean, Offshore and Arctic Engineering, 2011, 2011, rotterdam, Netherlands.

[16] M.-Y. Kim and Y.-U. Song, "The analysis of active power control requirements in the selected grid codes for wind farm," Journal of Electrical Engineering \& Technology, vol. 10(4), pp. 1409-1415, 2015.

[17] S. K. Salman, J. Gibb, and I. Macdonald, "Integration of tidal power based electrical plant into a grid," Universities Power Engineering Conference, UPEC 2008. 43 ${ }^{\text {rd }}$ International, 2008.

[18] M. Santos-Mugica, E. Robles, A. G. Endegnanew, E. Tedeschi, and J. Giebhardt, "Grid integration and power quality testing of marine energy converters: Research activities in the marinet project," in Ecological Vehicles and Renewable Energies (EVER), 2014 Ninth International Conference on. IEEE, 2014, pp. 1-9.

[19] A. D. Hansen, P. Sørensen, F. Iov, and F. Blaabjerg, "Centralised power control of wind farm with doubly fed induction generators," Renewable Energy, vol. 31, no. 7, pp. 935-951, 2006.

[20] A. K. Khamees, N. Badra, and A. Y. Abdelaziz, "Optimal power flow methods: A comprehensive survey."

[21] M. Alamir, M. Fiacchini, S. B. Chabane, S. Bacha, and T. Kovaltchouk, "Active power control under grid code constraints for a tidal farm," Eleventh International Conference on Ecological Vehicles and Renewable Energies (EVER), 2016,, 2016, monte Carlo.

[22] M. Alamir, "Lp-based identification of a general nonlinear multivariable maps: Preliminary results," hal-01231522, 2015, 2015.

[23] F. Ding and T. Chen, "Hierarchical least squares identification methods for multivariable systems," IEEE Transactions on Automatic Control, vol. 50(3), pp. 397-402, 2005.

[24] B.Bao, Y. Xu, J. Sheng, and F. Ding, "Least squares based iterative parameter estimation algorithm for multivariable controlled ARMA system modelling with finite measurement data," Mathematical and Computer Modelling, vol. 53(9-10), pp. 1664-1669, 2011.

[25] I. Mayung, "Tutorial on maximum likelihood estimation," Journal of Mathematical Psychology, vol. 47(1), pp. 90-100, 2003.

[26] J. Li, F. Ding, and G. Yang, "Maximum likelihood least squares identification method for input nonlinear finite impulse response moving average systems," Mathematical and Computer Modelling, vol. 55, pp. 442-450, 2012.

[27] M. Fiacchini, "Notes on wave-induced current predictors," GIPSA-lab, Research Report, 2015.

[28] RTE, "Documentation technique de référence, 2015," 2015.

[29] G. Chateigner, M. Boes, J. Chopin, and D. Verkindère, "Puissances, facteur de puissance et théorème de boucherot," Technologie, (2008), 158. 